\title{
LOS SABINARES ALBARES VALENCIANOS
}

\author{
Manuel COSTA, Rosa PEREZ-BADIA \& Pilar SORIANO
}

\begin{abstract}
RESUMEN: Se estudian los sabinares albares meso y supramediterráneos valencianos, su distribución, etapas seriales y estado de conservación.

Palabras clave: Vegetación, sabinares, Valencia, España.
\end{abstract}

SUMMARY: A study of the valencian mesomediterranean and supramediterranean Juniperus thurifera forest, their distribution, their substitution stages and their conservation state.

Key words: Vegetation, sabin grove, Valencia, Spain.

\section{N T R O D U C C I O N}

Los bosques sabineros (Juniperion thuriferae Rivas-Martínez 1969) son, sin duda, una de las formaciones arboladas más interesantes del Mediterráneo occidental. Su carácter relicto, así como sus condiciones ecológicas y corológicas, puestas de manifiesto por Rivas-Martínez (1969), son factores que realzan el valor de estos bosques y acrecientan el interés en su conservación.

Desde el estudio inicial de estas formaciones de sabina albar (Juniperus thurifera L.) por Rivas-Martínez (op. cit.), una serie de estudios posteriores en diferentes áreas han venido a completar aquella información y han permitido profundizar en su conocimiento facilitando los datos fitosociológicos, ecológicos y corológicos fundamentales para su conocimiento y conservación.

\section{LOS S A B INARESALBARES VALENCIANOS}

Actualmente, se reconocen en la Península Ibérica distintas series meso-supramediterráneas de sabina albar. Por un lado los sabinares meso supramediterráneos con carrascas (Junipero thuriferae-Quercetum rotundifoliae (Rivas Goday 1959) Rivas-Martínez 1987) de la clase Quercetea ilicis Br.-Bl. 1947, no obstante, es en la clase Pino-Juniperetea Rivas-Martínez 1964 donde se ubican la mayoría de los sabinares albares ibéricos, incluso el Juniperetum phoeniceo-thuriferae (Br.-Bl. \& O. Bolòs 1957) Rivas-Martínez 1987, quizás el más relacionado con la Quercetea ilicis- 


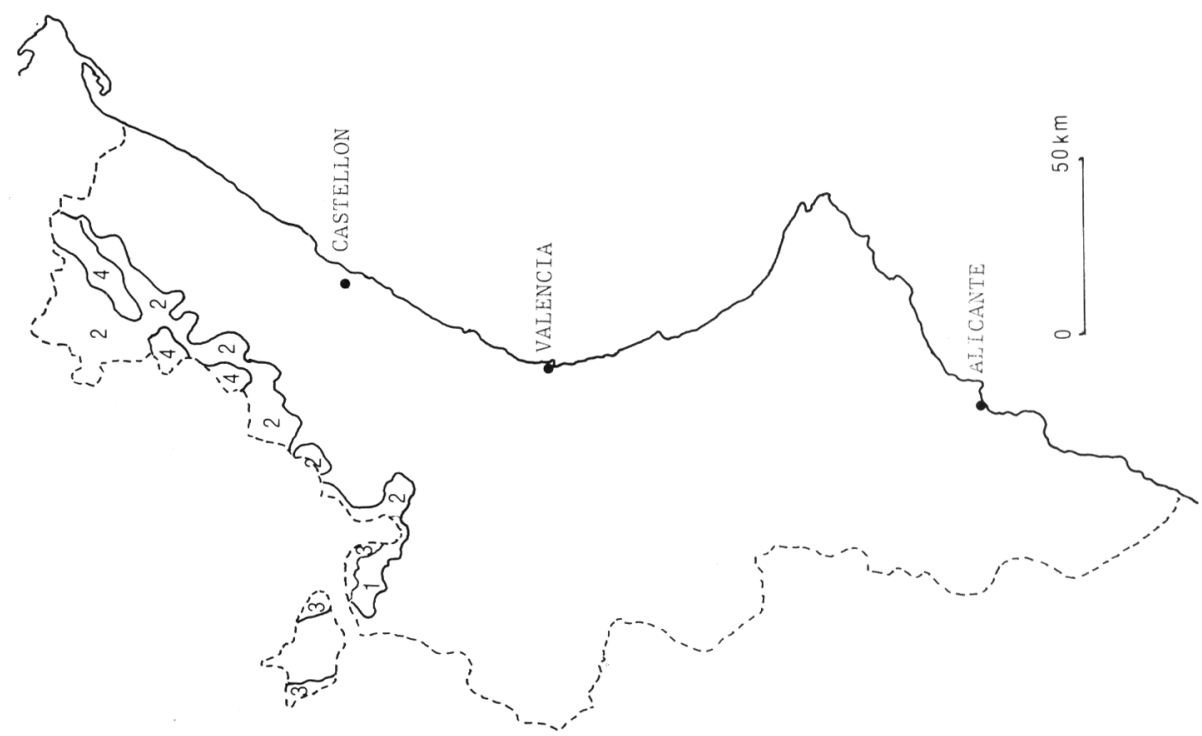

Distribución de la vegetación supramediterránea en el territorio valenciano: 1, Junipereto phoeniceae-thuriferae sigmetum. 2, Junipero thuriferae-Querceto rotundifoliae sigmetum. 3, Junipereto hemisphaerico-thuriferae sigmetum. 4, Violo-Querceto fagineae sigmetum.

Br.-B1. 1947. Por otro lado, los genuinos sabinares de la clase Pino-Juniperetea Rivas-Martínez 1964 (Juniperion thuriferae Rivas-Martínez 1969), entre los que cabe destacar los bosques supramediterráneos maestracenses y celtibérico-alcarreños del Juniperetum hemisphaerico-thuriferae Rivas-Martínez 1969 y los relictos eurosiberianos del Juniperetum sabino-thuriferae Rivas-Martínez, Izco \& Costa 1971, que para el piso montano orocantábrico describieron Rivas-Martínez \& al. (1971).

La situación del territorio valenciano, con tierras elevadas y frías en su interior, ya maestracenses o manchegas desde el punto de vista corológico, permite un buen desarrollo de los sabinares albares. En nuestro territorio, estas formaciones aún alcanzan grandes extensiones, sobre todo en los pisos mesomediterráneo superior y supramediterráneo entre las comarcas de Los Serranos y Els Ports, donde la serie del Junipero thuriferae-Querceto rotundifoliae sigmetum es la dominante. En algunos enclaves particulares, como sucede en La Yesa (Los Serranos), se reconoce la serie del Junipereto phoeniceo-thuriferae sigmetum como han puesto de manifiesto Costa (1986) y Rivas-Martínez (1987).

La mayoría de nuestros bosques sabineros están alterados. No obstante, el despoblamiento de estos duros territorios ha hecho, no sólo que se conserven algunos núcleos, sino además que se encuentren en proceso de franca recuperación.

Es en el Rincón de Ademuz donde se encuentran los sabinares mejor conservados. Estos bosques, muchos de ellos con un magnífico aspecto natural, se reparten por Sesga, La Puebla de San Miguel y el Cerro Calderón. En esta montaña, la más alta del País Valenciano $(1.839 \mathrm{~m})$, se puede reconocer una perfecta zonación desde el piso mesomediterráneo superior y supramediterráneo inferior con la serie $J u$ nipero thuriferae-Querceto rotundifoliae sigmetum, que da paso a la serie Junipereto hemisphaerico-thuriferae sigmetum de los pisos supramediterráneo medio y superior, 
para coronar a partir de $1.700 \mathrm{~m}$ con la serie Junipero sabinae-Pineto sylvestris sigmetum que se extiende ya por las cumbres.

Presentamos una tabla con once inventarios del Rincón de Ademuz y Los Serranos en Valencia, así como algunos de zonas colindantes de Teruel. En la tabla se puede apreciar una buena estratificación de la comunidad, encuadrable por su composición florística en el Juniperetum hemisphaerico thuriferae Rivas-Martínez 1969. En cuanto a la variabilidad, es muy frecuente la presencia de la subasociación de juniperetosum sabinae Rivas-Martínez 1969 (inv. 5 al 9) que matiza una faciación fría y de paso al Pino-Juniperion sabinae Rivas Goday (1956) 1960 oromediterráneo, este hecho ya fue puesto de manifiesto por Aguilella (1985). En los inventarios 10 y 11 puede reconocerse una variante de Juniperus phoenicea L. sobre suelos kársticos y denudados.

Todos estos sabinares, sobre todo los aclarados, alternan con tomillares pulvinulares de altura del Sideritido incanae-Salvion lavandulifoliae (Rivas Goday \& Rivas-Martínez 1969) Izco \& Molina 1984 y pastizales ralos del Festuco-Poion ligulatae Rivas Goday \& Rivas-Martínez 1963. De gran interés es el dinamismo de los sabinares sobre margas del valle de Torrijas a través de matorrales de Gypsophilion hispanicae Rivas Goday 1956, también puesto de manifiesto por Aguilella (op. cit.).

\section{O N S E R V A C I O N}

Los bosques sabineros valencianos, por su carácter relictual y su interés paisajístico, merecen una especial atención. En este sentido se deben intensificar las investigaciones sobre ellos para conocer sus características funcionales y poder vertebrar unas medidas adecuadas para su conservación basadas en una información científica y técnica adecuada.

Nota: Para Thymus godayanus seguimos el criterio de Rivas-Martínez, Molina \& Navarro (1988).

\section{B I B L I O G R A F I A}

AGUiLELLA, A. -1985-Flora y vegetación de la Sierra del Toro y Las Navas de Torrijas, "Estribaciones sudorientales del Macizo del Javalambre" Tesis doctoral Facultad de Biología. Universidad de Valencia.

COSTA, M. -1986- La vegetación en el País Valenciano. Servicio de Publicaciones, Universitat de Valencia.

RIVAS MARTINEZ, S. -1969- Vegetatio Hispanicae Notula I. Publ. Inst. Biol. Apl., 46:534. Barcelona.

RIVAS MARTINEZ, S. -1977- Memoria del Mapa de Series de Vegetación de España. I.C.O.N.A., Madrid.

RIVAS MARTINEZ, S., IZCO, J. \& COSTA, M. -1971- Sobre la flora y la vegetación del Macizo de Peña Ubiña. Trab. Dept. Botánica y Fisiol. Vegetal, 3:47-123. Madrid.

RIVAS MARTINEZ, S., MOLINA, A. \& NAVARRO, G. -1988- Nuevas especies del género Thymus sección Hyphodromi de la Península Ibérica. Opusc. Bot. Pharm. Complutensis, 4:107-121. Madrid.

(Aceptado para su publicación el 12 de febrero de 1990)

Dirección de los autores: Departament de Biología Vegetal (Botànica) Facultat de Farmàcia. Universitat de València. Blasco Ibañez, 13, 46010 València. 

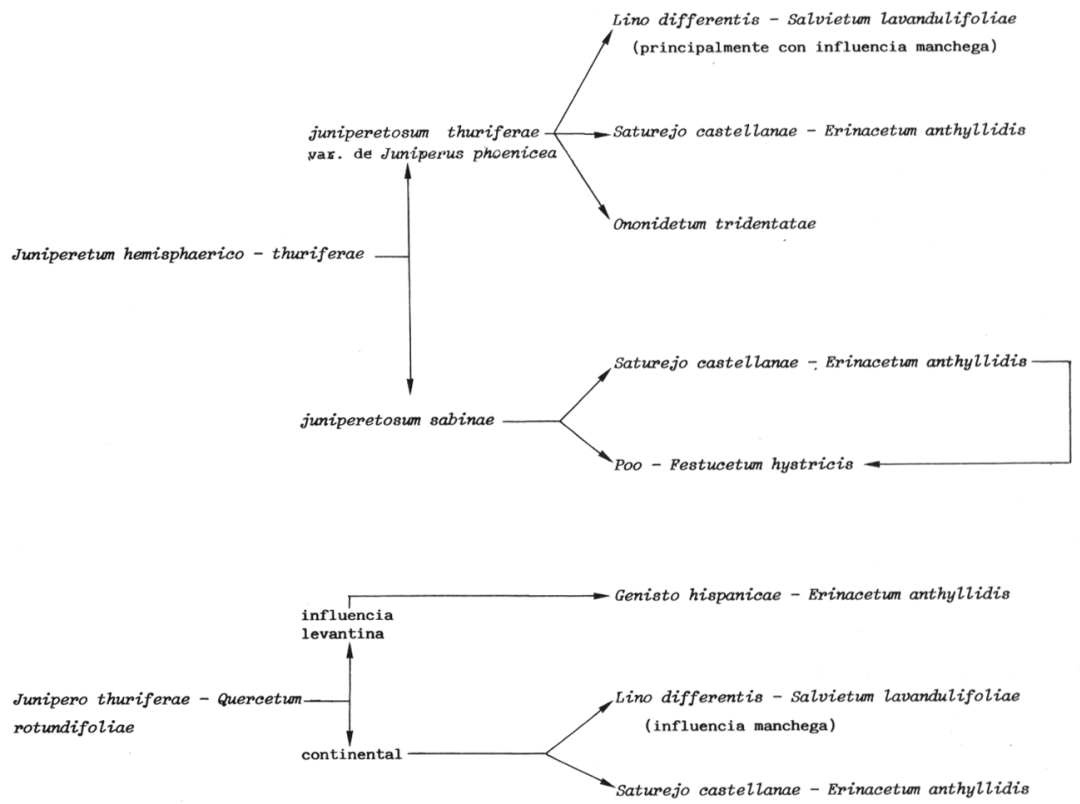

Dinamismo de los sabinares albares valencianos

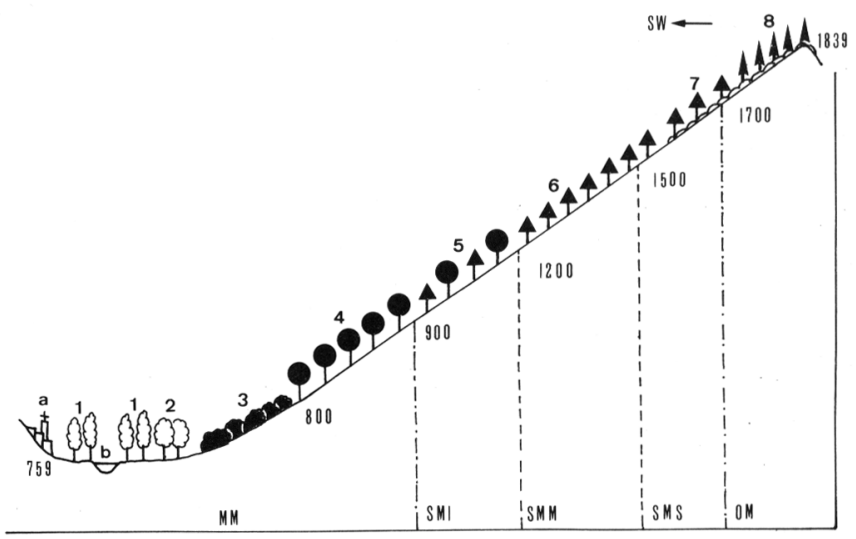

Zonación exquemática de la vegetación entre Ademuz y El Cerro Calderón: 1, Vinco-Populeto albae sigmetum. 2, Hedero-Ulmeto minoris sigmetum. 3, Rhamno lycioidis-Querceto rotundifoliae sigmetum. 4, Querceto rotundifoliae sigmetum. 5, Junipero thuriferaeQuerceto rotundifoliae sigmetum. 6, Junipereto hemisphaerico-thuriferae sigmetum. 7, Junipereto hemisphaerico thuriferae sigmetum, faciación de Juniperus sabina. 8, Junipero sabinae-Pineto sylvestris sigmetum. a, Ademuz. b, Río Turia. (MM: Mesomediterráneo, SMI: Supramediterráneo inferior, SMM: Supramediterráneo medio, SMS: Supramediterráneo superior, OM: Oromediterráneo). 


\section{Juniperetum hemisphaerico-thuriferae Rivas-Martínez 1969 juniperetosum sabinae Rivas-Martínez 1969 variante de Juniperus phoenicea}

\begin{tabular}{|c|c|c|c|c|c|c|c|c|c|c|}
\hline Area $\left(\mathrm{m}^{2}\right)$ & 100 & 80 & 100 & 70 & 100 & 70 & 100 & 100 & 90 & 70 \\
\hline Cobertura (\%) & 90 & 90 & 90 & 90 & 100 & 90 & 100 & 90 & 100 & 80 \\
\hline Altitud (m x 10) & 130 & 128 & 130 & 150 & 163 & 140 & 140 & 160 & 150 & 123 \\
\hline Exposición & NW & W & $\mathrm{N}$ & W & $\mathrm{NE}$ & SW & SW & $\mathrm{NE}$ & NW & SW \\
\hline Inclinación & 5 & 5 & 5 & 10 & 10 & 10 & 10 & 5 & - & 15 \\
\hline $\mathrm{N}^{\circ}$ de orden & 1 & 2 & 3 & 4 & 5 & 6 & 7 & 8 & 9 & 10 \\
\hline
\end{tabular}

Características de asociación y unidades superiores:

$\mathrm{E}_{1}$ :

$\begin{array}{lllllllllllll}\text { Jumiperus tharifera } & 2.3 & 2.2 & 2.2 & 2.3 & 1.2 & 2.3 & 2.3 & 2.2 & 1.2 & 2.2 & 1.1\end{array}$

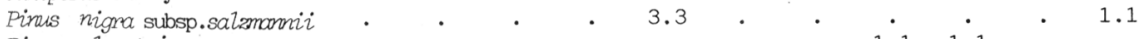

Pinas sylvestris

$\mathrm{E}_{2}$ :

Juniperus hemispharica $\quad \begin{array}{llllllllllllll}3.3 & 3.3 & 2.3 & 2.2 & 3.3 & 2.3 & 2.2 & 3.3 & 2.3 & 3.3 & 1.2\end{array}$

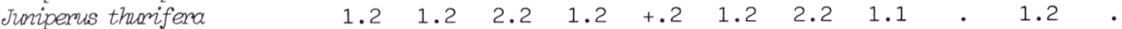

Diferenciales de subasociación y variante:

$\mathrm{E}_{2}$ :

Jroniperus sabina

Joniperus phoenicea

Jroniperus axycectrus

Compañeras:

$\mathrm{E}_{3}$ :

Thymus vrilgoaris

Genista scorprizs

Lavondula latifolia

Carex hallerana

silene legionensis

viola mpestris

sectum sediforme

Averaila bromoides

Potentilla tabernaemontani

scabiosa troolensis

Exphorbia nioaeensis

Erysimm grandiflorm

Helionthemm operminam

Leuisea conifena

Eryngirm compestre

Brachapodium retusm

Thymus godayaras

Koeleria vallesiona

\begin{tabular}{|c|c|c|c|c|c|c|c|c|c|c|}
\hline+.2 & 1.1 & 1.1 & 1.1 & 1.1 & 1.1 & 1.1 & 1.1 & 1.1 & 1.1 & 1.1 \\
\hline 1.2 & 1.1 & 2.2 & 1.2 & 1.1 & 2.2 & . & 1.1 & . & 2.2 & 2.2 \\
\hline+.2 & +.2 & 1.1 & +.2 & 1.2 & . & . & +.2 & +.2 & +.2 & 1.1 \\
\hline 1.2 & . & 1.2 & 2.2 & . & . & 1.2 & 1.1 & 2.2 & 1.2 & 1.1 \\
\hline 1.1 & 1.1 & . & 1.1 & . & 1.1 & - & . & - & - & •" \\
\hline . & . & 1.1 & 1.1 & 1.1 & - & . & 1.1 & 1.1 & 1.1 & 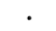 \\
\hline+ & + & 1.1 & +.2 & . & + & . & . & 1.1 & +.2 & 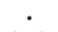 \\
\hline 1.2 & . & . & 1.2 & . & 2.2 & . & 1.1 & . & 1.2 & 1.1 \\
\hline+ & 1.1 & + & + & . & . & . & 1.1 & 1.1 & . & ${ }^{\circ}$ \\
\hline . & 1.1 & . & 1.1 & 1.1 & • & . & 1.1 & - & . & .1 \\
\hline 1.1 & 1.2 & + & . & . & . & . & . & 1.1 & . & \\
\hline$\cdot$ & 1.1 & . & . & - & 1.1 & . & 1.1 & 1.1 & . & \\
\hline . & 1.1 & 1.1 & 1.1 & + & . & . & . & . & . & 1. \\
\hline . & + & . & . & + & 1.1 & . & 1.1 & 1.1 & . & • \\
\hline . & . & . & . & + & + & 1.1 & . & 1.1 & - & + \\
\hline 2.2 & 1.2 & . & . & . & . & . & . & . & 3.3 & 1 \\
\hline . & . & . & . & 1.2 & 1.1 & . & 2.2 & 1.2 & - & \\
\hline . & . & . & 1.1 & 1.2 & . & . & . & - & 1.2 & \\
\hline
\end{tabular}

Además: en $\mathrm{E}_{2}$, compañeras: Quercus rotundifolia en 4, 1.2; Rhambus sascatilis en 7, 1.2; Rosmaminus officinalis en 4, 1.2; Rubia peregrina en 2, +.2. en $\mathrm{E}_{3}$, compañeras: Dactylis hispanica en $1,1.1$; en 2 , + y en 3, 1.1 ; Eminacea anthyllis en 3, +.2, en 6, 1.2 y en 11 1.1; Galizm fiuticescens en 4, 1.1, en 6, + y en 71.1 ; Helionthenrm rotiondifolizm en 1, 1.1, en 2, + y en 3, 1.2; Helianthemm caram en 4, +.2 y en 5, 1.1 ; Rosmorirus officinalis en 10, 1.1 y en 11, 3.3; Amshenatherm elatius en 7, 2.2 y en 11, 2.2; Teucrim chamaedris en $6,2.2$ y en $7,2.2$ y Rhamas sacatilis en $3,1.1$ y en $7,1.2$.

Localidades: 1, 2 y 3 Loma del Calvo, rincón de Ademuz (Valencia); 4, 5 y 8 subida al pico Caldenón, Rincón de Ademuz (Valencia); 6 Abejuela (Teruel); 7 Torrijas (Teruel); 9 Nava del Puerco, Abejuela (Teruel); 10 Hoya de la Carrasca (Teruel) y 11 Losilla de Aras-La Yesa (Valencia). 